\title{
Molecular mechanisms of natural killer cell activation in response to cellular stress
}

\author{
CJ Chan ${ }^{1,2,3}$, MJ Smyth ${ }^{\star, 1,2,3,4,5}$ and L Martinet ${ }^{1,2,4,5}$
}

Protection against cellular stress from various sources, such as nutritional, physical, pathogenic, or oncogenic, results in the induction of both intrinsic and extrinsic cellular protection mechanisms that collectively limit the damage these insults inflict on the host. The major extrinsic protection mechanism against cellular stress is the immune system. Indeed, it has been well described that cells that are stressed due to association with viral infection or early malignant transformation can be directly sensed by the immune system, particularly natural killer (NK) cells. Although the ability of NK cells to directly recognize and respond to stressed cells is well appreciated, the mechanisms and the breadth of cell-intrinsic responses that are intimately linked with their activation are only beginning to be uncovered. This review will provide a brief introduction to NK cells and the relevant receptors and ligands involved in direct responses to cellular stress. This will be followed by an in-depth discussion surrounding the various intrinsic responses to stress that can naturally engage NK cells, and how therapeutic agents may induce specific activation of NK cells and other innate immune cells by activating cellular responses to stress.

Cell Death and Differentiation (2014) 21, 5-14; doi:10.1038/cdd.2013.26; published online 12 April 2013

\section{Fact}

- Stress induces specific intrinsic and extrinsic physiological mechanisms within cells that lead to their identification as functionally abnormal

- Sources of cellular stress can be nutritional, physical, pathogenic, or oncogenic

- Intrinsic responses to cellular stress include activation of the DNA-damage response, tumor-suppressor genes, and senescence

- The extrinsic response to cellular stress is activation of the immune system, such as natural killer cells

- Intrinsic responses to cellular stress can directly upregulate factors that can activate the immune system, and the immune system been shown to be indispensable for the efficacy of some chemotherapy

\section{Open Questions}

- Further critical determinants of intrinsic responses to stress and cell death that can activate the immune system must be identified

- Identification of the different cellular pathways and molecular determinants controlling the immunogenicity of different cancer therapies is required
- How can we harness the ability of therapeutic agents to activate both the intrinsic and extrinsic responses to cellular stress to achieve more specific and safer approaches to cancer treatment?

\section{Cellular Stress and Natural Killer (NK) cells-An Overview}

Any insult to a cell that leads to its abnormal behavior or premature death can be defined as a source of stress. As the turnover and maintenance of cells in all multi-cellular organisms is tightly regulated, it is essential that stressed cells be rapidly identified to avoid widespread tissue damage and to maintain tissue homeostasis. Various intrinsic cellular mechanisms exist within cells that become activated when they are exposed to stress. These include activation of DNAdamage response proteins, senescence programs, and tumor-suppressor genes. ${ }^{1}$ Extrinsic mechanisms also exist that combat cellular stress, through the upregulation of mediators that can activate different components of the immune system. ${ }^{2}$ Although frequently discussed separately, much recent evidence has indicated that intrinsic and extrinsic responses to cellular stress are intimately linked. ${ }^{3}$

As the link between cell intrinsic and extrinsic responses to stress have been uncovered, these observations are now

\footnotetext{
${ }^{1}$ Cellular Immunity Laboratory, Cancer Immunology Program, Trescowthick Research Laboratories, Peter MacCallum Cancer Centre, East Melbourne, Australia; ${ }^{2}$ Sir Peter MacCallum Department of Oncology, University of Melbourne, Parkville, Australia; ${ }^{3}$ Department of Immunology, Monash University, AMREP, Prahran, Australia and ${ }^{4}$ Immunology of Cancer and Infection Laboratory, Queensland Institute of Medical Research, Herston, Australia

*Corresponding author: MJ Smyth, Immunology of Cancer and Infection Laboratory, Queensland Institute of Medical Research, 300 Herston Road, Herston 4006 , Australia. Tel: 0439300488 . Fax: 6173845 3032; E-mail: mark.smyth@ qimr.edu.au

${ }^{5}$ These authors contributed equally to this work.

Keywords: cellular stress; NK cells; therapy; cancer; immunogenic

Abbreviations: NK, natural killer; NKG2D, natural killer group 2D; DNAM-1, DNAX accessory molecule-1; MHC, major histocompatibility complex; MIC, MHC class I chain-related protein; ULBP, UL16-binding protein; Rae, retinoic acid early inducible; ATM, ataxia telangiectasia mutated; ATR, ATM and Rad3-related; miRNA, microRNA; HSP, heat-shock protein

Received 08.2.13; accepted 27.2.13; Edited by M Piacentini; published online 12.4.13
} 
being harnessed therapeutically, particularly in the context of cancer. ${ }^{4}$ Indeed, various chemotherapeutic agents and radiotherapy are critically dependent on the immune system to elicit their full therapeutic benefit. ${ }^{5,6}$ The mechanisms by which this occurs may be twofold: (i) the induction of intrinsic cellular stress mechanisms activates innate immunity and (ii) the release and presentation of tumor-specific antigens engages an inflammatory adaptive immune response.

NK cells are the major effector lymphocyte of innate immunity found in all the primary and secondary immune compartments as well as various mucosal tissues. ${ }^{7}$ Through their ability to induce direct cytotoxicity of target cells and produce pro-inflammatory cytokines such as interferongamma, NK cells are critically involved in the immune surveillance of tumors ${ }^{8,9,10}$ and microbial infections. ${ }^{11,12}$ The major mechanism that regulates NK cell contact-dependent functions (such as cytotoxicity and recognition of targets) is the relative contribution of inhibitory and activating receptors that bind to cognate ligands.

Under normal physiological conditions, NK cell activity is inhibited through the interaction of their inhibitory receptors with major histocompatibility complex (MHC) class I. ${ }^{13,14}$ However, upon instances of cellular stress that are frequently associated with viral infection and malignant transformation, ligands for activating receptors are often upregulated and MHC class I expression may be downregulated. The upregulation of these activating ligands and downregulation of MHC class I thus provides a signal for NK cells to become activated and display effector functions. Activating receptors are able to provide NK cells with a strong stimulus in the absence of co-stimulation due to the presence of adaptor molecules such as DAP10, DAP12, $\mathrm{FcR} \gamma$, and $\mathrm{CD} 3 \zeta$ that contain immunoreceptor tyrosine-based activating motifs (ITAMs). ${ }^{15-17}$ By contrast, inhibitory receptors contain inhibitory motifs (ITIMs) within their cytoplasmic tails that can activate downstream targets such as SHP-1 and SHP-2 and directly antagonize those signaling pathways activated through ITAMs. ${ }^{18-20}$ The specific details of individual classes of inhibitory and activating receptors and their ligands are summarized in Figure 1 and have been extensively reviewed elsewhere. ${ }^{14,21}$ Instead, this review will more focus on the relevant activating receptors that are primarily involved in the direct regulation of NK cell-mediated recognition of cellular stress: natural killer group 2D (NKG2D) and DNAX accessory molecule-1 (DNAM-1).

\section{NK Cell-Mediated Recognition of Cellular Stress by NKG2D and DNAM-1}

NKG2D is a lectin-like type 2 transmembrane receptor expressed as a homodimer in both mice and humans by virtually all NK cells. ${ }^{22,23}$ Upon interaction with its ligands, NKG2D can trigger NK cell-mediated cytotoxicity against their targets. The ligands for NKG2D are self proteins related to MHC class I molecules. ${ }^{24}$ In humans, these ligands consist of the $\mathrm{MHC}$ class I chain-related protein (MIC) family (e.g., MICA and $\mathrm{MICB}$ ) and the UL16-binding protein (ULBP1-6) family. ${ }^{25,26}$ In mice, ligands for NKG2D include the retinoic acid early inducible (Rae) gene family, the H60 family, and mouse ULBP-like transcript-1 (MULT-1). ${ }^{27-29}$ NKG2D ligands are generally absent on the cell surface of healthy cells but are frequently upregulated upon cellular stress associated with viral infection and malignant transformation. ${ }^{3,30}$ Indeed, NKG2D ligand expression has been found on many transformed cell lines, and NKG2D-dependent elimination of tumor cells expressing NKG2D ligands has been well documented in vitro and in tumor transplant experiments. ${ }^{25,30-33}$ In humans, NKG2D ligands have been described on different primary tumors ${ }^{34,35}$ and specific NKG2D gene polymorphisms are associated with susceptibility to cancer. ${ }^{36}$ Finally, blocking NKG2D through gene inactivation or monoclonal antibodies leads to an increased susceptibility to tumor development in mouse models, ${ }^{37,38}$ demonstrating the key role played by NKG2D in immune surveillance of tumors. NKG2D can also contribute to shape tumor immunogenicity, a process called immunoediting, as demonstrated by the frequent ability of tumor cells to avoid NKG2D-mediated recognition through NKG2D ligand shedding, as discussed later in this review. ${ }^{38-40}$

DNAM-1 is a transmembrane adhesion molecule constitutively expressed on T cells, NK cells, macrophages, and a small subset of $B$ cells in mice and humans. ${ }^{41-43}$ DNAM-1 contains an extracellular region with two IgV-like domains, a transmembrane region and a cytoplasmic region containing tyrosine- and serine-phosphorylated sites that is able to initiate downstream activation cascades. ${ }^{41,44}$ There is accumulating evidence showing that DNAM-1 not only promotes adhesion of NK cells and CTLs but also greatly enhances their cytotoxicity toward ligand-expressing targets. ${ }^{41,45-50}$ The ligands for DNAM-1 are the nectin/nectin-like family members CD155 (PVR, necl-5) and CD112 (PVRL2, nectin-2). ${ }^{45,46}$ Like NKG2D ligands, DNAM-1 ligands are frequently expressed on virus-infected and transformed cells. ${ }^{51,52}$ DNAM-1 ligands, especially CD155, are overexpressed by many types of solid and hematological malignancies and blocking DNAM-1 interactions with its ligands reduces the ability of NK cells to kill tumor cells in vitro. ${ }^{41,49,53-57}$ Further evidence of the role of DNAM-1 in tumor immune surveillance is provided by studies using experimental and spontaneous models of cancer in vivo showing enhanced tumor spread in the absence of DNAM-1. ${ }^{47-50,58}$

As NKG2D and DNAM-1 ligands are frequently expressed on stressed cells, many studies have sought to determine the mechanisms that underpin these observations. The guiding hypothesis for these studies is that cell-intrinsic responses to stress are directly linked to cell-extrinsic responses that can trigger rapid NK cell surveillance and elimination of stressed cells. Indeed, major cell-intrinsic responses to cellular stress can directly lead to NK cell-activating ligand upregulation and are outlined in the following sections.

\section{The DNA-Damage Response}

Cellular stress caused by the activation of the DNA-damage response leads to downstream apoptosis or cell-cycle arrest. The activation of DNA-damage checkpoints occurs when there are excessive DNA strand breaks and replication errors, thereby representing an important tumorigenesis barrier that can slow or inhibit the progression of malignant transformation. ${ }^{59,60}$ Two major transducers of the DNAdamage response are the PI3-kinase-related protein kinases 


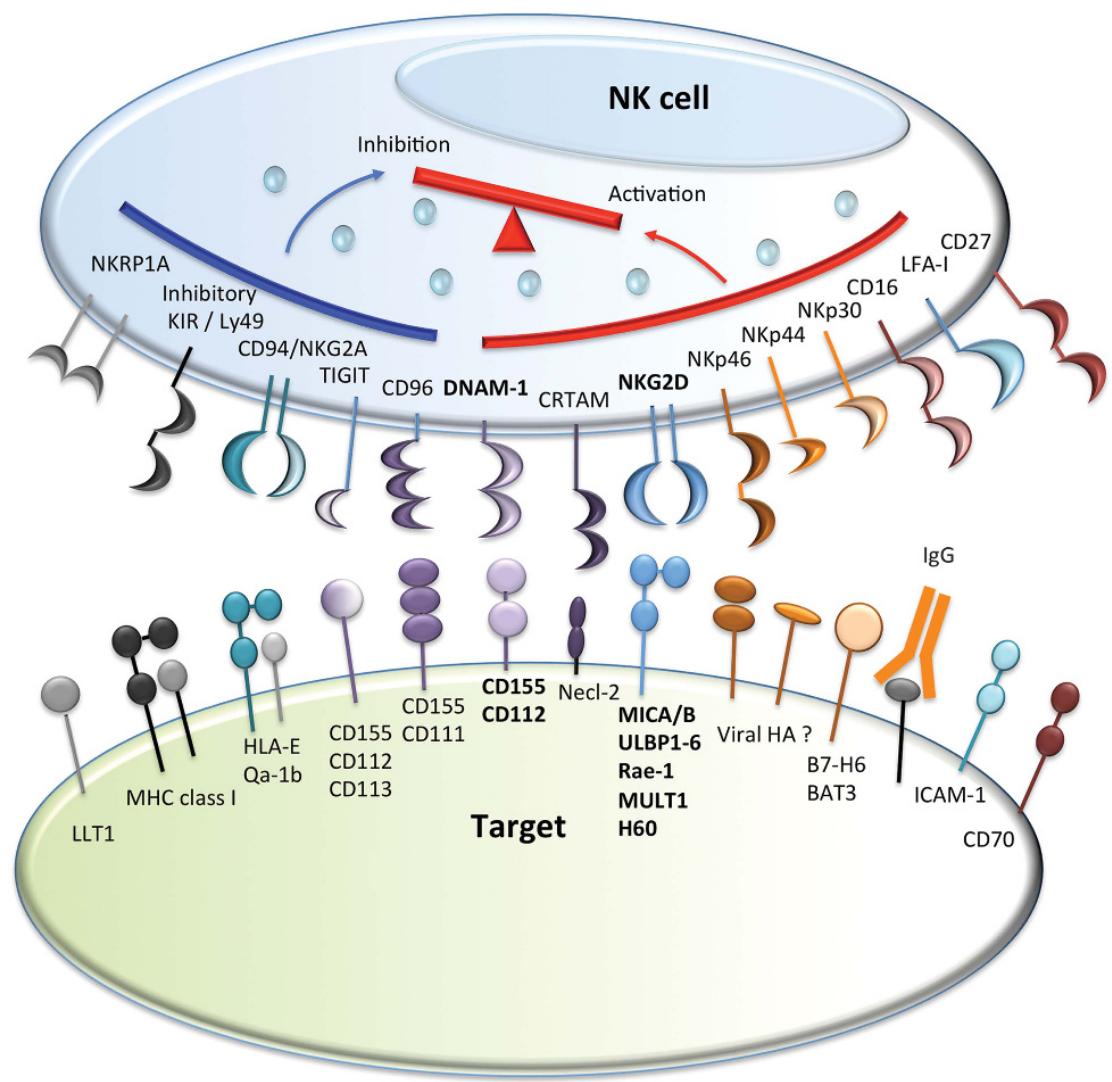

Figure 1 NK cell receptors and their cognate ligands. Major inhibitory and activating receptors on NK cells and their cognate ligands on targets are depicted. BAT3, human leukocyte antigen (HLA)-B-associated transcript 3; CRTAM, class I-restricted T-cell-associated molecule; HA, hemagglutinin; HLA-E, HLA class I histocompatibility antigen, alpha chain E; IgG, immunoglobulin G; LFA-1, leukocyte function-associated antigen-1; LLT1, lectin-like transcript 1; TIGIT, T cell immunoglobulin and ITIM domain

ATM (ataxia telangiectasia mutated) and ATR (ATM and Rad3-related). ATM and ATR can modulate numerous signaling pathways such as checkpoint kinases (Chk1 and Chk2, which inhibit cell-cycle progression and promote DNA repair) and p53 (which mediates cell-cycle arrest and apoptosis). ${ }^{61}$

In addition to the induction of cell-cycle arrest and apoptosis, activation of the DNA-damage response has been shown to promote the expression of several activating ligands that are specific for NK cell receptors, primarily those of the NKG2D receptor. These findings have shown a critical direct link between cellular transformation, apoptosis, and surveillance by the immune system. ${ }^{62}$ The first evidence of this link between DNA damage and immune cell activation was provided by Raulet and colleagues who showed that NKG2D ligands were upregulated by genotoxic stress and stalled DNA replication conditions known to activate either ATM or ATR. ${ }^{63}$ These observations have now been extended by several other studies that have defined further DNA-damaging conditions (e.g., genotoxic drugs/chemotherapy, deregulated proliferation, or oxidative stress) that can promote NKG2D ligand upregulation. ${ }^{64-67}$

The role of the DNA-damage response in controlling NKG2D ligand expression and subsequent NK cell activation has also been demonstrated in the context of anti-viral immunity, specifically in Abelson murine leukemia virus infection. ${ }^{68}$ This pathogen was shown to induce activationinduced cytidine deaminase (AID) expression outside the germinal center, resulting in generalized hypermutation, DNAdamage checkpoint activation, and Chk1 phosphorylation. The genotoxic activity of virally induced AID not only restricted the proliferation of infected cells but also induced the expression of NKG2D ligands. More recently, another member of APOBEC-AID family of cytidine deaminases, A3G, has been shown to promote the recognition of HIVinfected cells by NK cells after DNA-damage response activation. ${ }^{69}$ In this study, viral protein Vpr-mediated repair processes, which generate nicks, gaps, and breaks of DNA, activate an ATM/ATR DNA-damage response that leads to NKG2D ligand expression.

The DNA-damage sensors ATM and ATR have also been shown to regulate other key NK cell-activating ligands such as the DNAM-1 ligand, CD155. ${ }^{58,65,70}$ For example, in the $\mathrm{E} \mu$-myc spontaneous B-cell lymphoma model, activation of the DNA-damage response leads to the upregulation of CD155 in the early-stage transformed B cells, subsequently activating spontaneous tumor regression in an NK cell- and T-cell-dependent manner. ${ }^{58}$ The DNA-damage response can also regulate the expression of the death receptor DR5 ${ }^{71}$ The engagement of DR5 by the effector molecule TRAIL, which is expressed by NK cells and T cells, can induce apoptosis of target cells and has been shown to have a key role in immune 


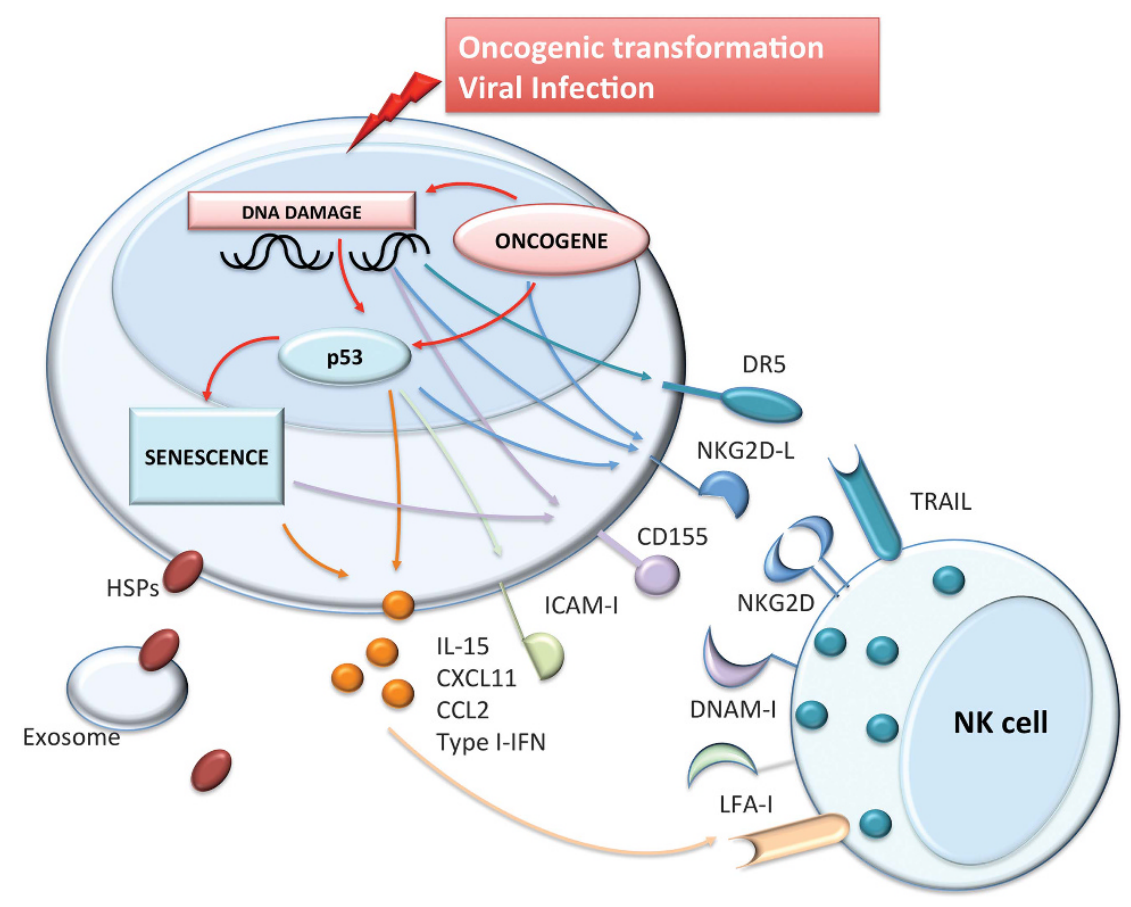

Figure 2 Overview of the molecular pathways leading to NK cell recognition of intrinsic cellular stress. Oncogenic transformation and viral infection can activate intrinsic cellular responses to stress. These responses include activation of the DNA-damage response, senescence, tumor suppressors, and the presentation and/or release of HSPs that, in turn, can activate NK cells through various receptor-ligand interactions. Senescent cells can also release pro-inflammatory cytokines that can recruit NK cells and other innate immunity, such as macrophages. CCL2, C-C motif chemokine ligand 2; CXCL11, C-X-C motif chemokine ligand 11; DR, death receptor 5; IFN, interferon; IL, interleukin; LFA-1, leukocyte function-associated antigen-1; TRAIL, tumor necrosis factor-related apoptosis-inducing ligand

surveillance against tumors. ${ }^{72}$ Collectively, these results suggest that the detection of DNA damage, primarily through ATM and ATR, may represent a conserved protection mechanism governing the immunogenicity of infected or transformed cells, leading to direct recognition by NK cells (Figure 2).

As a result of these studies, many therapeutic agents known to induce DNA damage have been evaluated for their ability to increase the immunogenicity of cancer cells for a more targeted therapeutic approach using NK cells. ${ }^{64,65}$ For example, treatment of multiple myeloma cells with doxorubicin, melphalan, or bortezomib can lead to DNAM-1 and NKG2D ligand upregulation. ${ }^{65}$ Indeed, many chemotherapeutic agents commonly used, especially in hematological malignancies, can trigger the DNA-damage pathway. Therefore, it is reasonable to speculate that there is a general role of ATM and ATR in the induction of NK cell activation as a therapeutic effect of these agents.

\section{Senescence}

Cellular senescence is generally defined as a growth-arrest program in mammalian cells that limits their lifespan. ${ }^{73}$ The major type of cellular senescence is replicative senescence that occurs due to telomere shortening. However, it is now generally accepted that premature senescence can also occur due to oncogene activation (oncogene-induced senescence) and/or the loss/gain of tumor-suppressor gene function, in the absence of telomere shortening. ${ }^{74}$ Thus, premature senescence is an important barrier against malignant transformation. ${ }^{59}$ Upon engagement of the senescence program, although cells are in growth arrest, they remain metabolically active and can produce many proinflammatory cytokines, as well as upregulate adhesion molecules and activating ligands to alert the immune system. ${ }^{75-77}$ Activation of the immune system, in particular innate immunity, has a critical role in the clearance of senescent cells. ${ }^{78-81}$ More specifically, in a model of hepatocellular carcinoma, it has been shown that reactivation of p53 can induce a senescence program, resulting in tumor regression through the activation of NK cells, macrophages, and neutrophils. Of note, intercellular adhesion molecule (ICAM)-1, which can trigger both adhesion and cytotoxicity of NK cells, ${ }^{82}$ and interleukin-15, a cytokine that can promote NK cell effector function, ${ }^{83}$ were both upregulated in senescent tumors. More recently, the potential contribution of NK cells was also shown in the clearance of senescent hepatic stellate cells, a mechanism important in limiting liver fibrosis in response to a fibrogenic agent. ${ }^{80}$ ICAM-1, NKG2D ligands (MICA and ULPB2), and DNAM-1 ligands (CD155) were all upregulated on senescent hepatic stellate cells.

The specific mechanisms linking the senescence program to immune activation are not yet fully understood. However, the intracellular molecular mechanisms that govern induction of senescence may provide possible indications. Both replicative senescence and premature senescence (e.g., oncogene-induced senescence) have been shown to have common molecular determinants, such as the activation of the 
DNA-damage response pathway (e.g., ATM and ATR) and downstream activation of $p 53$ and $p 16^{\text {INK4A }}$ 1,59,84-86 $^{\text {Activa- }}$ tion of the DNA-damage response would presumably initiate the upregulation of NK cell-activating ligands as previously discussed. However, how senescence may be linked to the induction of pro-inflammatory cytokine release is a more compelling question and requires further investigation (Figure 2). Nevertheless, induction of pro-inflammatory cytokines is an important protective mechanism in order to recruit immune cells that can rapidly recognize and remove senescent cells. Interestingly, activation of NK cells by senescent cells has been observed in a clinical context when multiple myeloma cells were treated with chemotherapy and genotoxic agents. ${ }^{65}$ In this setting, NKG2D and DNAM-1 ligands were both upregulated through a mechanism that required activation of the DNA-damage pathway initiated by ATM and ATR. ${ }^{65}$

\section{Tumor Suppressors: p53}

p53 is a potent tumor suppressor and central regulator of apoptosis, DNA repair, and cell proliferation, that is activated in response to DNA damage, oncogene activation, and other cellular stress. ${ }^{87}$ The number of identified cellular functions that $\mathrm{p} 53$ regulates has greatly increased over the past few years, and there is now a vast array of evidence that shows that $p 53$ can be induced by viral infection ${ }^{88}$ to limit pathogen spread by inducing apoptosis. ${ }^{89,90}$ Furthermore, p53 not only acts as an intrinsic barrier against tumorigenesis or pathogenic spread but can also lead to increased cellular immunogenicity. For example, p53 reactivation in a hepatocellular carcinoma can promote tumor regression mediated by innate immunity. ${ }^{78} \mathrm{~A}$ direct link between p53 expression and immune cell recognition was recently provided by Textor et al. $^{91}$ where expression of p53 in lung cancer cell lines strongly upregulated the NKG2D ligands ULBP1 and 2, resulting in NK cell activation. Subsequently, p53-responsive elements were found to directly regulate ULBP1 and 2 expression, the deletion of which abolished the capacity of p53 to mediate ULBP1 and 2 upregulation. Another recent report that used a pharmacological activator of $p 53$ confirmed the ability of p53 to directly induce ULBP2 expression that was independent of ATM/ATR. ${ }^{92}$ However, it has also been shown that miR34a and miR34C microRNAs (miRNAs) induced by p53 can target ULBP2 mRNA and reduce its cell-surface expression, suggesting that p53 may have a dual role in regulating ULBP2 expression. ${ }^{93}$ Finally, early work showed that NKG2D ligands can be upregulated by ATR/ATM in the total absence of $p 53$ in tumor cell lines, ${ }^{62,63}$ suggesting the existence of ATM/ATR-dependent and p53-independent pathways that regulate NKG2D ligand expression in response to cellular stress.

In addition to regulating NK cell ligand expression, genetic reactivation of $p 53$ in tumors can also induce a wide array of pro-inflammatory mediators ranging from adhesion receptor (ICAM-1) expression to the production of various chemokines (CXCL11 and monocyte chemoattractant protein-1) and cytokines (interleukin-15). ${ }^{78}$ Furthermore, recent studies in anti-viral immunity indicate that several interferon-inducible genes and Toll-like receptor-3 expression are direct transcriptional targets of p53 and that p53 contributes to production of type I interferon by virally infected cells. ${ }^{94-96}$ All together, these studies suggest that p53 accumulation could represent a key determinant of the immunogenicity of stressed cells that are infected or undergoing malignant transformation through its ability to regulate innate immune activation.

\section{Oncogenes}

Malignant transformation is a complex process that frequently involves the activation of one or more oncogenes in addition to the inactivation or mutation of tumor-suppressor genes (e.g., p53). Oncogene activation is a powerful inducer of cellular stress that is able to activate intrinsic cellular programs that lead to cell apoptosis or senescence (e.g., activation of the DNA-damage response and p53). ${ }^{1}$ In addition, many recent reports have also shown that major oncogenes can activate extrinsic responses to cellular stress through inducing the upregulation of NK cell-activating ligands. ${ }^{63,97,98}$ This suggests that oncogene activation can represent a key cellular event in alerting the immune system to ongoing cellular transformation (Figure 3).

The enhanced expression of the proto-oncogene Myc has been described as a critical event leading to cellular transformation and is a frequently found genetic alteration in cancer. ${ }^{99}$ In a recent study, again using the $\mathrm{E} \mu$-myc model, Medzhitov and colleagues demonstrated the ability of c-Myc to alert NK cells to early oncogenic transformation through the upregulation of Rae-1. ${ }^{97}$ In this study, the induction of Rae-1 was dependent on the direct regulation of Rae-1 transcription by Myc through its interaction with the Raet1 epsilon gene. Collectively, these results provide a possible direct molecular mechanism to explain the increased susceptibility of NKG2D gene-targeted mice to lymphoma development in the $\mathrm{E} \mu$-myc model. ${ }^{38}$

Recent evidence suggests that several oncogenic mutations of Ras (H-Ras, N-Ras, and K-Ras) can also regulate NKG2D ligand expression in both mice and humans. ${ }^{98}$ Interestingly, in this case, NKG2D ligands were regulated through MAPK/MEK and PI3K pathways downstream of oncogenic H-RasV12. The activation of PI3K pathways, and more particularly the $\mathrm{p} 110 \alpha$ subunits by virus-encoded proteins, has also been shown to induce the Rae-1 family of ligands. ${ }^{100}$ As many viruses can manipulate the PI3K pathway $^{101}$ and tumors often bear Ras and $\mathrm{p} 110 \alpha$ oncogene mutations, ${ }^{102}$ collectively, this data suggests that there is the existence of a common molecular mechanism by which NK cells sense cellular stress mediated by PI3K-dependent regulation of NKG2D ligands.

Interestingly, whereas Myc was involved in the transcriptional regulation of NKG2D ligands, PI3K can increase NKG2D ligand expression by increasing the translation of Rae-1 mRNA. ${ }^{98}$ This involved the induction of elF4E, a protein that enhances the translation of mRNA. ${ }^{103}$ As number of tumors and viruses can upregulate host translation initiation machinery through the overexpression of elF4E, ${ }^{104,105}$ this may represent an important means by which NK cells can discriminate tumor- and virus-infected cells from normal cells. 


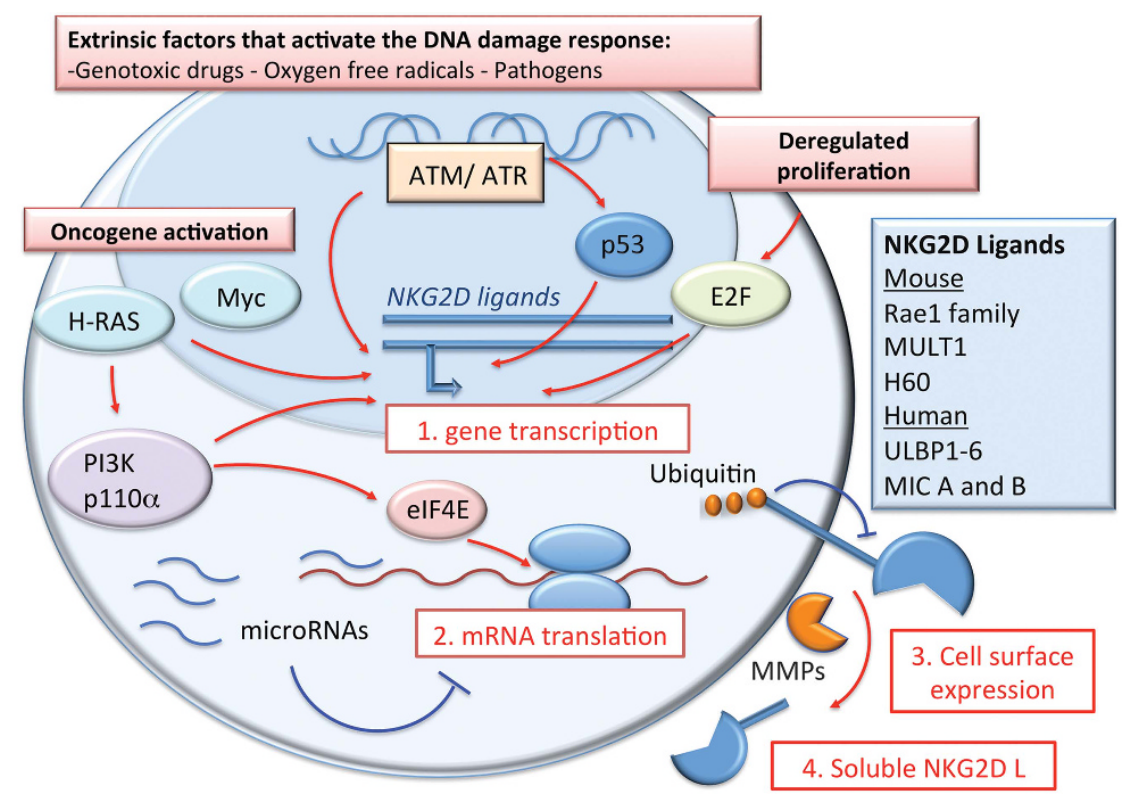

Figure 3 Molecular mechanisms that regulate the cell surface expression of NKG2D ligands. The major group of NK cell-activating ligands that are upregulated by intrinsic cellular responses to stress are those that bind the NKG2D receptor. Activation of the DNA-damage response, senescence, oncogenes, tumor suppressors, or sensing of deregulated proliferation can induce NKG2D ligand gene transcription and increase mRNA translation, leading to extracellular protein expression. MMP, matrix metalloproteases

\section{Heat-Shock Proteins (HSPs)}

HSPs are highly conserved intracellular chaperone molecules that are present in most prokaryotic and eukaryotic cells that mediate protection against cellular damage under conditions of stress. HSPs are distributed in most intracellular compartments of cells where they support the correct folding of nascent polypeptides, prevent protein aggregation, and assist in protein transport across membranes. ${ }^{106}$ Many tumors display overexpression of HSPs as a response to cellular stress induced by oncogenic transformation. ${ }^{107,108}$ HSPs can also be mobilized to the plasma membrane, or even released from cells, under conditions of stress. ${ }^{109}$

Although intracellular HSPs can promote cell survival by interfering with different apoptosis components, many studies have reported that membrane-bound or soluble HSPs can directly stimulate innate immunity. ${ }^{110} \mathrm{~A}$ major immunostimulatory function of HSPs is to promote the presentation of tumorspecific antigens by MHC class I to CD8 T cells. ${ }^{111-113}$ Soluble and membrane-bound HSPs can also induce antigen-presenting cell maturation and the resultant secretion of pro-inflammatory cytokines. ${ }^{114-116}$ Finally, HSPs may directly activate NK cells as HSP70, when overexpressed on tumor cells, can induce a selective dose-dependent increase in NK cellmediated cytotoxicity in vitro. ${ }^{117} \mathrm{NK}$ cells may directly recognize HSP70 through a 14-amino-acid oligomer (TKD) that is localized in the C-terminal domain of the protein through CD94. ${ }^{118,119}$ Tumor-specific HSP70 that is either presented at the cell surface or secreted on exosomes can also enhance NK cell activity against diverse types of cancer in vivo. ${ }^{120,121}$ Most importantly, hepatocellular carcinoma cells that are treated with various chemotherapeutic agents can become more susceptible to NK cell-mediated cytotoxicity through their release of HSP-containing exosomes, giving the aforementioned findings a therapeutic context. ${ }^{122}$ Collectively, these results suggest that HSP translocation to the plasma membrane or secretion during cellular stress may represent a potent danger signal that can stimulate NK cell activity, particularly in the context of cancer.

\section{Emerging Links Between Cellular Stress and NK Cell Activation}

Deregulated proliferation. Deregulated proliferation is a critical determinant of cellular transformation and pathogenic infection. ${ }^{123,124}$ Interestingly, recent results indicate that the Rae-1 family of ligands is a transcriptional target for E2F transcription factors, which control cell-cycle progression. ${ }^{25}$ Cellular stress induced by deregulated proliferation may therefore activate NKG2D-bearing lymphocytes, including NK cells, as an early barrier to tumorigenesis. Future studies will have to address whether E2F transcription factors or indeed other cell-cycle regulators can also modulate other NK cell-activating ligands and whether these mechanisms can promote NK cell-tumor immunosurveillance in vivo.

MiRNAs. MiRNAs are an abundant class of small noncoding RNAs that are approximately 22 nucleotides in length. The functions of miRNAs are to directly regulate proteinencoding mRNAs to suppress gene expression. ${ }^{126}$ Owing to their ability to regulate gene expression, miRNAs have been implicated in many biological and pathological processes. ${ }^{127}$ Recent studies demonstrate that several miRNAs can regulate the cell-surface expression of NKG2D ligands (MICA, MICB, and ULBP2), therefore implicating miRNAs as a link between intrinsic and extrinsic responses to oncogenic transformation. ${ }^{93,128,129}$ Indeed, in the context of cancer, many miRNAs (designated oncomiRs) are activated and are involved in the initiation and progression of 
disease. ${ }^{130}$ As such, several oncomiRs have been implicated in the suppression of NKG2D ligand upregulation in various types of cancer, leading to their ability to facilitate tumor escape from NK cell-mediated immunosurveillance. ${ }^{93,129}$

Regulation of cell-surface expression. In addition to transcriptional and post-transcriptional regulation of NK cell receptor ligand expression by intrinsic cellular pathways, recent evidence suggest that stress-induced mechanisms can affect cell-surface expression of such ligands. For example, the expression of MULT-1 NKG2D ligand is repressed at the protein level on normal cells by polyubiquitination of its cytoplasmic tail leading to its degradation. By contrast, upon cellular stress induced by UV irradiation or heat shock, MULT-1 is less ubiquitinated resulting in an increase in cell-surface expression. ${ }^{131}$

NKG2D ligand shedding, secretion, or excretion in vesicles such as exosomes also seems to regulate their level of expression at the cell surface. For example, cleavage of the extracellular domain of NKG2D ligands by matrix metalloproteases results in their shedding from the cell surface, a common escape mechanism used by tumor cells to avoid NK cell recognition. ${ }^{39,40,132,133}$ Indeed, soluble NKG2D ligands have been described in many cancer types in humans and usually correlates with a negative prognosis. ${ }^{40,133-135}$ Therefore, a better understanding of the intrinsic and extrinsic molecular pathways implicated in NKG2D ligand shedding could have direct therapeutic implications.

Cellular hyperploidy. Cellular hyperploidy occurs when there is an acquisition of more than two sets of chromosomes. For example, tetraploidy (or tetraploidization), where there is the presence of four sets of chromosomes, has been associated with early tumorigenesis due the acquisition of extra genetic material inherently making cells more susceptible to genetic abnormalities. As a barrier to potential oncogenic transformation from cellular hyperploidy, this event can be intrinsically sensed, leading to the inhibition of cell-cycle progression. ${ }^{136}$ In a recent report, it has also been shown that cellular hyperploidy can be extrinsically sensed by the immune system. ${ }^{137}$ Hyperploid cells were shown to become immunogenic as a result of a constitutive endoplasmic reticulum stress response that leads to the cell-surface exposure of calreticulin, a potent phagocytic signal for antigen-presenting cells, such as macrophages. ${ }^{138}$ Interestingly, in this study, it was also shown that ex vivo tumors from $\mathrm{E} \mu$-myc mice crossed to a DNAM-1 ${ }^{-1}$

background exhibited a larger nuclear diameter and P-elF2 $\alpha$ phosphorylation (as surrogate markers for cellular hyperploidy) than tumors from a wild-type background. This suggests that DNAM-1 on NK cells (and T cells) may participate in the immunoselection of hyperploid cells.

Immunogenic cell death. There is now clear evidence that demonstrates that the full clinical efficacy of some conventional chemo- and radiotherapy requires the activation of several immune components. ${ }^{139,140}$ A common determinant of this requirement is the ability of these therapies to induce immunogenic cancer cell death. More specifically, it has been shown that cell-surface exposure of calreticulin and the release of high mobility group box-1 and adenosine triphosphate can activate dendritic cells leading to engulfment of dying cells, tumor-specific antigen presentation, and production of pro-inflammatory cytokines such as interleu$\operatorname{kin}-1 \beta^{6,141,142}$ (Figure 4). Interestingly, although CD8 T cells were the main effectors responsible for the therapeutic effects of chemotherapy in these settings, the efficient priming of these cells required the presence of upstream innate immunity, such as $\gamma \delta$ T cells. ${ }^{143}$ As the phenotype and

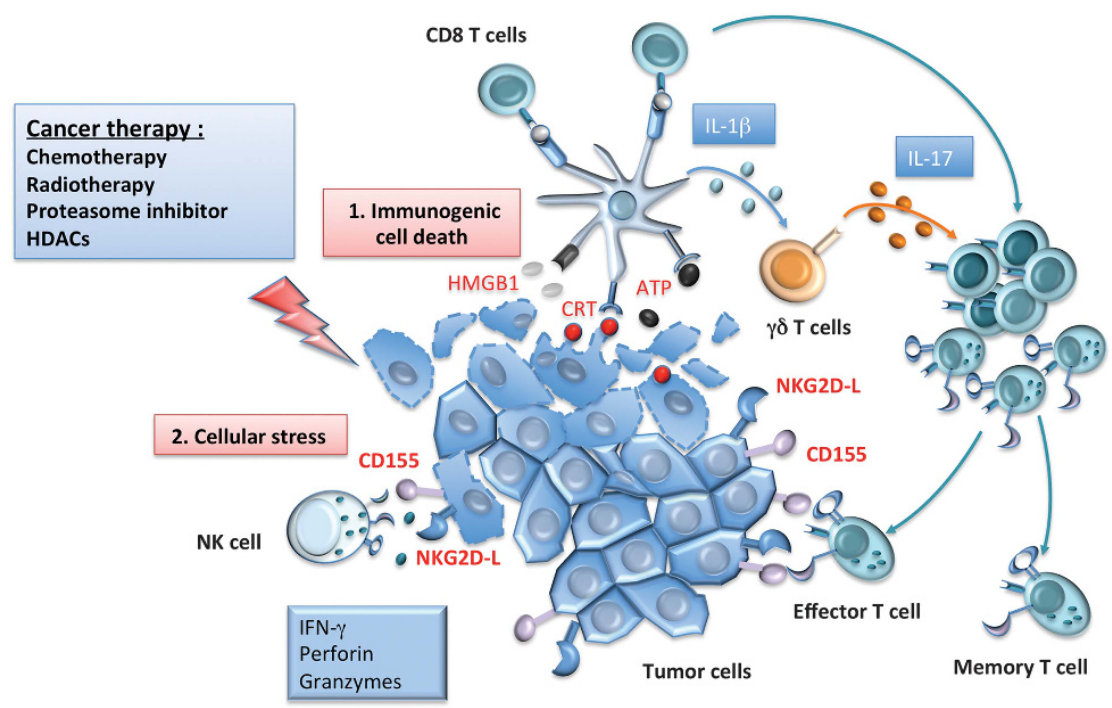

Figure 4 The role of NK cells during immunogenic cell death induced by cancer therapy. (1) Immunogenic cell death induced by various cancer therapies such as chemotherapy and radiotherapy involves the cell surface exposure of calreticulin (CRT), and the release of high mobility group box (HMGB)-1 and adenosine triphosphate (ATP) that triggers dendritic cell engulfment of dying cells, antigen presentation, and production of interleukin (IL)-1 $\beta$, ultimately leading to activation of CD8 T cells. The efficient priming of tumor-specific CD8 T cells may require the presence of innate immunity such as $\gamma \delta$ T cells that produce IL-17 in response to IL-1 $\beta$. (2) Various cancer therapies can also activate intrinsic cellular responses to stress in tumor cells that can result in the upregulation of NKG2D ligands and the DNAM-1 ligand, CD155. This subsequently leads to the recognition and destruction of tumors by NK cells and CD8 T cells. HDAC, histone deacetylases; IFN, interferon 
function of NK cells are modulated in humans during chemotherapy $^{144,145}$ and many cellular stress pathways activated by chemotherapy can increase tumor cell sensitivity to NK cell-mediated cytotoxicity and recognition (as discussed in the previous sections), it will be important to determine whether NK cells can also contribute to the efficacy of chemotherapy.

\section{Concluding Remarks}

NK cells provide an important primary defense mechanism against stressed cells. This is due to their ability to respond rapidly to the upregulation of multiple activating ligands. Although frequently documented on stressed cells, the mechanisms that govern the regulation of NK cell-activating ligand expression have only recently become apparent, particularly in the context of cancer. These mechanisms include multiple intrinsic cellular responses, such as the activation of the DNA-damage response, senescence, the activation of tumor suppressors and oncogenes, and the sensing of deregulated cell proliferation. Indeed, many forms of chemo- and radiotherapy that are currently used in the clinic that aim to engage these intrinsic cellular responses have been shown to require the immune system, including NK cells for their full efficacy. Thus, formulating specific therapies that can concurrently engage intrinsic and extrinsic responses to cellular stress will hopefully lead to more targeted, effective, and safer approaches for the treatment of cancer.

\section{Conflict of Interest}

The authors declare no conflict of interest.

Acknowledgements. CJC is supported by a Leukaemia Foundation of Australia PhD scholarship. MJS and LM are supported by a NHMRC Australia Fellowship, Program, and Project Grant.

1. Lowe SW, Cepero E, Evan G. Intrinsic tumour suppression. Nature 2004; 432: 307-315.

2. Griffith TS, Ferguson TA. Cell death in the maintenance and abrogation of tolerance: the five Ws of dying cells. Immunity 2011; 35: 456-466.

3. Raulet DH, Guerra N. Oncogenic stress sensed by the immune system: role of natural killer cell receptors. Nat Rev 2009; 9: 568-580.

4. Kroemer G, Galluzzi L, Kepp O, Zitvogel L. Immunogenic cell death in cancer therapy. Annu Rev Immunol 2013; 31: 51-72.

5. Denkert C, Loibl S, Noske A, Roller M, Muller BM, Komor M et al. Tumor-associated lymphocytes as an independent predictor of response to neoadjuvant chemotherapy in breast cancer. J Clin Oncol 2010; 28: 105-113.

6. Apetoh L, Ghiringhelli F, Tesniere A, Obeid M, Ortiz C, Criollo A et al. Toll-like receptor 4-dependent contribution of the immune system to anticancer chemotherapy and radiotherapy. Nat Med 2007; 13: 1050-1059.

7. Vivier E, Tomasello E, Baratin M, Walzer T, Ugolini S. Functions of natural killer cells. Nat Immunol 2008; 9: 503-510.

8. Smyth MJ, Thia KY, Cretney E, Kelly JM, Snook MB, Forbes CA et al. Perforin is a major contributor to NK cell control of tumor metastasis. J Immunol 1999; 162: 6658-6662.

9. Smyth MJ, Thia KY, Street SE, Cretney E, Trapani JA, Taniguchi M et al. Differential tumor surveillance by natural killer (NK) and NKT cells. J Exp Med 2000; 191: 661-668.

10. Smyth MJ, Crowe NY, Godfrey DI. NK cells and NKT cells collaborate in host protection from methylcholanthrene-induced fibrosarcoma. Int Immunol 2001; 13: 459-463.

11. Andoniou CE, Andrews DM, Degli-Esposti MA. Natural killer cells in viral infection: more than just killers. Immunol Rev 2006; 214: 239-250.

12. Andrews DM, Scalzo AA, Yokoyama WM, Smyth MJ, Degli-Esposti MA. Functional interactions between dendritic cells and NK cells during viral infection. Nat Immuno/ 2003; 4: $175-181$.

13. Raulet DH, Vance RE. Self-tolerance of natural killer cells. Nat Rev 2006; 6: 520-531.

14. Karre K. Natural killer cell recognition of missing self. Nat Immunol 2008; 9: 477-480.
15. Gilfillan S, Ho EL, Cella M, Yokoyama WM, Colonna M. NKG2D recruits two distinct adapters to trigger NK cell activation and costimulation. Nat Immunol 2002; 3: 1150-1155.

16. Vivier E, Nunes JA, Vely F. Natural killer cell signaling pathways. Science 2004; 306 : 1517-1519.

17. Campbell JL, Klueva NY, Zheng HG, Nieto-Sotelo J, Ho TD, Nguyen HT. Cloning of new members of heat shock protein HSP101 gene family in wheat (Triticum aestivum (L.) Moench) inducible by heat, dehydration, and ABA(1). Biochim Biophys Acta 2001; 1517: 270-277.

18. Purdy AK, Campbell KS. SHP-2 expression negatively regulates NK cell function. $\mathrm{J}$ Immunol 2009; 183: 7234-7243.

19. Yusa S, Catina TL, Campbell KS. SHP-1- and phosphotyrosine-independent inhibitory signaling by a killer cell Ig-like receptor cytoplasmic domain in human NK cells. J Immunol 2002; 168: 5047-5057.

20. Yusa S, Campbell KS. Src homology region 2-containing protein tyrosine phosphatase-2 (SHP-2) can play a direct role in the inhibitory function of killer cell Ig-like receptors in human NK cells. J Immunol 2003; 170: 4539-4547.

21. Lanier LL. Up on the tightrope: natural killer cell activation and inhibition. Nat Immunol 2008; 9: 495-502.

22. Raulet DH. Roles of the NKG2D immunoreceptor and its ligands. Nat Rev 2003; 3: 781-790.

23. Champsaur M, Lanier LL. Effect of NKG2D ligand expression on host immune responses. Immunol Rev 2010; 235: 267-285.

24. Raulet DH, Gasser S, Gowen BG, Deng W, Jung H. Regulation of Ligands for the NKG2D Activating Receptor. Annu Rev Immunol 2013; 31: 413-441

25. Bauer S, Groh V, Wu J, Steinle A, Phillips JH, Lanier LL et al. Activation of NK cells and T cells by NKG2D, a receptor for stress-inducible MICA. Science 1999; 285 : 727-729.

26. Cosman D, Mullberg J, Sutherland CL, Chin W, Armitage R, Fanslow W et al. ULBPs, novel MHC class I-related molecules, bind to CMV glycoprotein UL16 and stimulate NK cytotoxicity through the NKG2D receptor. Immunity 2001; 14: 123-133.

27. Cerwenka A, Bakker AB, McClanahan T, Wagner J, Wu J, Phillips JH et al. Retinoic acid early inducible genes define a ligand family for the activating NKG2D receptor in mice. Immunity 2000; 12: 721-727.

28. Carayannopoulos LN, Naidenko OV, Fremont DH, Yokoyama WM. Cutting edge: murine UL16-binding protein-like transcript 1: a newly described transcript encoding a highaffinity ligand for murine NKG2D. J Immunol 2002; 169: 4079-4083.

29. Diefenbach A, Jamieson AM, Liu SD, Shastri N, Raulet DH. Ligands for the murine NKG2D receptor: expression by tumor cells and activation of NK cells and macrophages. Nat Immunol 2000; 1: 119-126.

30. Cerwenka A, Lanier LL. Natural killer cells, viruses and cancer. Nat Rev 2001; 1: 41-49.

31. Pende D, Cantoni C, Rivera P, Vitale M, Castriconi R, Marcenaro S et al. Role of NKG2D in tumor cell lysis mediated by human NK cells: cooperation with natural cytotoxicity receptors and capability of recognizing tumors of nonepithelial origin. Eur $\mathrm{J}$ Immunol 2001; 31: 1076-1086.

32. Diefenbach A, Jensen ER, Jamieson AM, Raulet DH. Rae1 and $\mathrm{H} 60$ ligands of the NKG2D receptor stimulate tumour immunity. Nature 2001; 413: 165-171.

33. Jamieson AM, Diefenbach A, McMahon CW, Xiong N, Carlyle JR, Raulet DH. The role of the NKG2D immunoreceptor in immune cell activation and natural killing. Immunity 2002; 17: 19-29.

34. Groh V, Rhinehart R, Secrist H, Bauer S, Grabstein KH, Spies T. Broad tumor-associated expression and recognition by tumor-derived gamma delta T cells of MICA and MICB. Proc Natl Acad Sci USA 1999; 96: 6879-6884.

35. Wu JD, Higgins LM, Steinle A, Cosman D, Haugk K, Plymate SR. Prevalent expression of the immunostimulatory $\mathrm{MHC}$ class I chain-related molecule is counteracted by shedding in prostate cancer. J Clin Investig 2004; 114: 560-568.

36. Hayashi T, Imai K, Morishita Y, Hayashi I, Kusunoki Y, Nakachi K. Identification of the NKG2D haplotypes associated with natural cytotoxic activity of peripheral blood lymphocytes and cancer immunosurveillance. Cancer Res 2006; 66: 563-570.

37. Smyth MJ, Swann J, Cretney E, Zerafa N, Yokoyama WM, Hayakawa Y. NKG2D function protects the host from tumor initiation. J Exp Med 2005; 202: 583-588.

38. Guerra N, Tan YX, Joncker NT, Choy A, Gallardo F, Xiong N et al. NKG2D-deficient mice are defective in tumor surveillance in models of spontaneous malignancy. Immunity 2008; 28: $571-580$.

39. Groh V, Wu J, Yee C, Spies T. Tumour-derived soluble MIC ligands impair expression of NKG2D and T-cell activation. Nature 2002; 419: 734-738.

40. Jinushi M, Vanneman M, Munshi NC, Tai YT, Prabhala RH, Ritz J et al. MHC class I chain-related protein $\mathrm{A}$ antibodies and shedding are associated with the progression of multiple myeloma. Proc Natl Acad Sci USA 2008; 105: 1285-1290.

41. Shibuya A, Campbell D, Hannum C, Yssel H, Franz-Bacon K, McClanahan T et al. DNAM-1, a novel adhesion molecule involved in the cytolytic function of T lymphocytes. Immunity 1996; 4: 573-581.

42. Fuchs $A$, Colonna M. The role of NK cell recognition of nectin and nectin-like proteins in tumor immunosurveillance. Semin Cancer Biol 2006; 16: 359-366.

43. Chan CJ, Andrews DM, Smyth MJ. Receptors that interact with nectin and nectin-like proteins in the immunosurveillance and immunotherapy of cancer. Curr Opin Immunol 2012; 24: 246-251. 
44. Sherrington PD, Scott JL, Jin B, Simmons D, Dorahy DJ, Lloyd J et al. TLiSA1 (PTA1) activation antigen implicated in T cell differentiation and platelet activation is a member of the immunoglobulin superfamily exhibiting distinctive regulation of expression. J Biol Chem 1997; 272: 21735-21744.

45. Bottino C, Castriconi R, Pende D, Rivera P, Nanni M, Carnemolla B et al. Identification of PVR (CD155) and Nectin-2 (CD112) as cell surface ligands for the human DNAM-1 (CD226) activating molecule. J Exp Med 2003; 198: 557-567.

46. Pende D, Spaggiari GM, Marcenaro S, Martini S, Rivera P, Capobianco A et al. Analysis of the receptor-ligand interactions in the natural killer-mediated lysis of freshly isolated myeloid or lymphoblastic leukemias: evidence for the involvement of the Poliovirus receptor (CD155) and Nectin-2 (CD112). Blood 2005; 105: 2066-2073.

47. Gilfillan S, Chan CJ, Cella M, Haynes NM, Rapaport AS, Boles KS et al. DNAM-1 promotes activation of cytotoxic lymphocytes by nonprofessional antigen-presenting cells and tumors. J Exp Med 2008; 205: 2965-2973.

48. Iguchi-Manaka A, Kai H, Yamashita Y, Shibata K, Tahara-Hanaoka S, Honda S et al. Accelerated tumor growth in mice deficient in DNAM-1 receptor. J Exp Med 2008; 205 2959-2964.

49. Lakshmikanth T, Burke S, Ali TH, Kimpfler S, Ursini F, Ruggeri L et al. NCRs and DNAM-1 mediate NK cell recognition and lysis of human and mouse melanoma cell lines in vitro and in vivo. J Clin Investig 2009; 119: 1251-1263.

50. Chan CJ, Andrews DM, McLaughlin NM, Yagita H, Gilfillan S, Colonna M et al. DNAM-1/ CD155 interactions promote cytokine and NK cell-mediated suppression of poorly immunogenic melanoma metastases. J Immunol 2010; 184: 902-911.

51. Tomasec P, Wang EC, Davison AJ, Vojtesek B, Armstrong M, Griffin C et al. Downregulation of natural killer cell-activating ligand CD155 by human cytomegalovirus UL141. Nat Immunol 2005; 6: 181-188.

52. Morisaki T, Onishi H, Katano M. Cancer immunotherapy using NKG2D and DNAM-1 systems. Anticancer Res 2012; 32: 2241-2247.

53. Pende D, Bottino C, Castriconi R, Cantoni C, Marcenaro S, Rivera P et al. PVR (CD155) and Nectin-2 (CD112) as ligands of the human DNAM-1 (CD226) activating receptor: involvement in tumor cell lysis. Mol Immunol 2005; 42: 463-469.

54. El-Sherbiny YM, Meade JL, Holmes TD, McGonagle D, Mackie SL, Morgan AW et al. The requirement for DNAM-1, NKG2D, and NKp46 in the natural killer cell-mediated killing of myeloma cells. Cancer Res 2007; 67: 8444-8449.

55. Cho D, Shook DR, Shimasaki N, Chang YH, Fujisaki H, Campana D. Cytotoxicity of activated natural killer cells against pediatric solid tumors. Clin Cancer Res 2010; 16 3901-3909

56. Mamessier E, Sylvain A, Bertucci F, Castellano R, Finetti $P$, Houvenaeghel $G$ et al. Human breast tumor cells induce self-tolerance mechanisms to avoid NKG2D-mediated and DNAM-mediated NK cell recognition. Cancer Res 2011; 71: 6621-6632.

57. Mamessier E, Sylvain A, Thibult ML, Houvenaeghel G, Jacquemier J, Castellano R et al. Human breast cancer cells enhance self tolerance by promoting evasion from NK cell antitumor immunity. J Clin Investig 2011; 121: 3609-3622.

58. Croxford JL, Tang ML, Pan MF, Huang CW, Kamran N, Phua CM et al. ATM-dependent spontaneous regression of early Emu-myc-induced murine $B$ cell leukemia depends on NK and T cells. Blood 2013; 121: 2512-2521.

59. Bartkova J, Rezaei N, Liontos M, Karakaidos P, Kletsas D, Issaeva N et al. Oncogeneinduced senescence is part of the tumorigenesis barrier imposed by DNA damage checkpoints. Nature 2006; 444: 633-637.

60. Gorgoulis VG, Vassiliou LV, Karakaidos P, Zacharatos P, Kotsinas A, Liloglou T et al. Activation of the DNA damage checkpoint and genomic instability in human precancerous lesions. Nature 2005; 434: 907-913.

61. Shiloh Y. ATM and related protein kinases: safeguarding genome integrity. Nat Rev Cancer 2003; 3: 155-168.

62. Gasser S, Raulet DH. The DNA damage response arouses the immune system. Cance Res 2006; 66: 3959-3962.

63. Gasser S, Orsulic S, Brown EJ, Raulet DH. The DNA damage pathway regulates innate immune system ligands of the NKG2D receptor. Nature 2005; 436: 1186-1190.

64. Vales-Gomez M, Chisholm SE, Cassady-Cain RL, Roda-Navarro P, Reyburn HT. Selective induction of expression of a ligand for the NKG2D receptor by proteasome inhibitors. Cancer Res 2008; 68: 1546-1554.

65. Soriani A, Zingoni A, Cerboni C, lannitto ML, Ricciardi MR, Di Gialleonardo V et al. ATMATR-dependent up-regulation of DNAM-1 and NKG2D ligands on multiple myeloma cells by therapeutic agents results in enhanced NK-cell susceptibility and is associated with a senescent phenotype. Blood 2009; 113: 3503-3511.

66. Berghuis D, Schilham MW, Vos HI, Santos SJ, Kloess S, Buddingh EP et al. Histone deacetylase inhibitors enhance expression of NKG2D ligands in Ewing sarcoma and sensitize for natural killer cell-mediated cytolysis. Clin Sarcoma Res 2012; 2: 8.

67. Cerboni C, Zingoni A, Cippitelli M, Piccoli M, Frati L, Santoni A. Antigen-activated human T lymphocytes express cell-surface NKG2D ligands via an ATM/ATR-dependent mechanism and become susceptible to autologous NK- cell lysis. Blood 2007; 110: 606-615

68. Gourzi P, Leonova T, Papavasiliou FN. A role for activation-induced cytidine deaminase in the host response against a transforming retrovirus. Immunity 2006; 24: 779-786.

69. Norman JM, Mashiba M, McNamara LA, Onafuwa-Nuga A, Chiari-Fort E, Shen W et al. The antiviral factor APOBEC3G enhances the recognition of HIV-infected primary T cells by natural killer cells. Nat Immunol 2011; 12: 975-983.
70. Ardolino M, Zingoni A, Cerboni C, Cecere F, Soriani A, lannitto ML et al. DNAM-1 ligand expression on Ag-stimulated T lymphocytes is mediated by ROS-dependent activation of DNA-damage response: relevance for NK-T cell interaction. Blood 2011; 117: 4778-4786.

71. Wu GS, Burns TF, McDonald ER 3rd, Jiang W, Meng R, Krantz ID et al. KILLER/DR5 is a DNA damage-inducible p53-regulated death receptor gene. Nat Genet 1997; 17: $141-143$.

72. Johnstone RW, Frew AJ, Smyth MJ. The TRAIL apoptotic pathway in cancer onset, progression and therapy. Nat Rev Cancer 2008; 8: 782-798.

73. Narita M, Lowe SW. Senescence comes of age. Nat Med 2005; 11: 920-922.

74. Kuilman T, Michaloglou C, Mooi WJ, Peeper DS. The essence of senescence. Genes Dev 2010; 24: 2463-2479.

75. Kuilman T, Michaloglou C, Vredeveld LC, Douma S, van Doorn R, Desmet CJ et al. Oncogene-induced senescence relayed by an interleukin-dependent inflammatory network. Cell 2008; 133: 1019-1031.

76. Acosta JC, O'Loghlen A, Banito A, Guijarro MV, Augert A, Raguz S et al. Chemokine signaling via the CXCR2 receptor reinforces senescence. Cell 2008; 133: 1006-1018.

77. Krizhanovsky V, Xue W, Zender L, Yon M, Hernando E, Lowe SW. Implications of cellular senescence in tissue damage response, tumor suppression, and stem cell biology. Cold Spring Harb Symp Quant Biol 2008; 73: 513-522.

78. Xue W, Zender L, Miething C, Dickins RA, Hernando E, Krizhanovsky V et al. Senescence and tumour clearance is triggered by p53 restoration in murine liver carcinomas. Nature 2007; 445: 656-660.

79. Sagiv A, Biran A, Yon M, Simon J, Lowe SW, Krizhanovsky V. Granule exocytosis mediates immune surveillance of senescent cells. Oncogene 2012; e-pub ahead of print 2 July 2012; doi:10.1038/onc.2012.206.

80. Krizhanovsky V, Yon M, Dickins RA, Hearn S, Simon J, Miething C et al. Senescence of activated stellate cells limits liver fibrosis. Cell 2008; 134: 657-667.

81. Wall M, Poortinga G, Stanley KL, Lindemann RK, Bots M, Chan CJ et al. The mTORC1 inhibitor Everolimus prevents and treats Emu-Myc lymphoma by restoring oncogeneinduced senescence. Cancer Discov 2013; 3: 82-95.

82. Poggi A, Pardi R, Pella N, Morelli L, Sivori S, Vitale M et al. CD45-mediated regulation of LFA1 function in human natural killer cells. Anti-CD45 monoclonal antibodies inhibit the calcium mobilization induced via LFA1 molecules. Eur J Immunol 1993; 23: 2454-2463.

83. Lucas M, Schachterle W, Oberle K, Aichele P, Diefenbach A. Dendritic cells prime natural killer cells by trans-presenting interleukin 15. Immunity 2007; 26: 503-517.

84. d'Adda di Fagagna F, Reaper PM, Clay-Farrace L, Fiegler H, Carr P, Von Zglinicki T et al. A DNA damage checkpoint response in telomere-initiated senescence. Nature 2003; 426 : 194-198.

85. Serrano M, Lin AW, McCurrach ME, Beach D, Lowe SW. Oncogenic ras provokes premature cell senescence associated with accumulation of p53 and p16INK4a. Cell 1997; 88: 593-602.

86. Di Micco R, Fumagalli M, Cicalese A, Piccinin S, Gasparini P, Luise C et al. Oncogeneinduced senescence is a DNA damage response triggered by DNA hyper-replication. Nature 2006; 444: 638-642.

87. Green DR, Kroemer G. Cytoplasmic functions of the tumour suppressor p53. Nature 2009; 458: 1127-1130.

88. Rivas C, Aaronson SA, Munoz-Fontela C. Dual Role of p53 in Innate Antiviral Immunity. Viruses 2010; 2: 298-313.

89. Munoz-Fontela C, Garcia MA, Garcia-Cao I, Collado M, Arroyo J, Esteban M et al. Resistance to viral infection of super p53 mice. Oncogene 2005; 24: 3059-3062.

90. Turpin E, Luke K, Jones J, Tumpey T, Konan K, Schultz-Cherry S. Influenza virus infection increases p53 activity: role of p53 in cell death and viral replication. $J$ Virol 2005; 79: 8802-8811.

91. Textor S, Fiegler N, Arnold A, Porgador A, Hofmann TG, Cerwenka A. Human NK cells are alerted to induction of $p 53$ in cancer cells by upregulation of the NKG2D ligands ULBP1 and ULBP2. Cancer Res 2011; 71: 5998-6009.

92. Li H, Lakshmikanth T, Garofalo C, Enge M, Spinnler C, Anichini A et al. Pharmacological activation of $p 53$ triggers anticancer innate immune response through induction of ULBP2. Cell Cycle 2011; 10: 3346-3358.

93. Heinemann A, Zhao F, Pechlivanis S, Eberle J, Steinle A, Diederichs S et al. Tumor suppressive microRNAs miR-34a/c control cancer cell expression of ULBP2, a stress-induced ligand of the natural killer cell receptor NKG2D. Cancer Res 2012; 72: 460-471

94. Munoz-Fontela C, Macip S, Martinez-Sobrido L, Brown L, Ashour J, Garcia-Sastre A et al. Transcriptional role of p53 in interferon-mediated antiviral immunity. J Exp Med 2008; 205: 1929-1938.

95. Imbeault M, Ouellet M, Tremblay MJ. Microarray study reveals that HIV-1 induces rapid type-I interferon-dependent p53 mRNA up-regulation in human primary CD4 $+\mathrm{T}$ cells. Retrovirology 2009; 6 : 5 .

96. Taura M, Eguma A, Suico MA, Shuto T, Koga T, Komatsu K et al. p53 regulates Toll-like receptor 3 expression and function in human epithelial cell lines. Mol Cell Biol 2008; 28: $6557-6567$

97. Unni AM, Bondar T, Medzhitov R. Intrinsic sensor of oncogenic transformation induces a signal for innate immunosurveillance. Proc Natl Acad Sci USA 2008; 105: 1686-1691.

98. Liu XV, Ho SS, Tan JJ, Kamran N, Gasser S. Ras activation induces expression of Raet1 family NK receptor ligands. J Immunol 2012; 189: 1826-1834. 
99. Dang CV. MYC on the path to cancer. Cell 2012; 149: 22-35.

100. Tokuyama M, Lorin C, Delebecque F, Jung H, Raulet DH, Coscoy L. Expression of the RAE-1 family of stimulatory NK-cell ligands requires activation of the PI3K pathway during viral infection and transformation. PLoS Pathogens 2011; 7: e1002265.

101. Buchkovich NJ, Yu Y, Zampieri CA, Alwine JC. The TORrid affairs of viruses: effects of mammalian DNA viruses on the PI3K-Akt-mTOR signalling pathway. Nat Rev Microbiol 2008; 6: 266-275.

102. Zhao L, Vogt PK, Class I. PI3K in oncogenic cellular transformation. Oncogene 2008; 27 : 5486-5496.

103. Mamane Y, Petroulakis E, Rong L, Yoshida K, Ler LW, Sonenberg N. elF4E-from translation to transformation. Oncogene 2004; 23: 3172-3179.

104. Ruggero D. The role of Myc-induced protein synthesis in cancer. Cancer Res 2009; 69: 8839-8843.

105. Walsh D. Manipulation of the host translation initiation complex elF4F by DNA viruses. Biochem Soc Trans 2010; 38: 1511-1516.

106. Hartl FU, Hayer-Hartl M. Molecular chaperones in the cytosol: from nascent chain to folded protein. Science 2002; 295: 1852-1858.

107. Jolly C, Morimoto RI. Role of the heat shock response and molecular chaperones in oncogenesis and cell death. J Natl Cancer Inst 2000; 92: 1564-1572.

108. Evans CG, Chang L, Gestwicki JE. Heat shock protein 70 (hsp70) as an emerging drug target. J Med Chem 2010; 53: 4585-4602.

109. Johnson JD, Fleshner M. Releasing signals, secretory pathways, and immune function of endogenous extracellular heat shock protein 72. J Leukoc Biol 2006; 79: 425-434.

110. Srivastava P. Roles of heat-shock proteins in innate and adaptive immunity. Nat Rev 2002; 2: 185-194.

111. Udono $\mathrm{H}$, Srivastava PK. Heat shock protein 70 -associated peptides elicit specific cancer immunity. J Exp Med 1993; 178: 1391-1396.

112. Arnold-Schild D, Hanau D, Spehner D, Schmid C, Rammensee HG, de la Salle $H$ et al. Cutting edge: receptor-mediated endocytosis of heat shock proteins by professional antigen-presenting cells. J Immunol 1999; 162: 3757-3760.

113. Srivastava P. Interaction of heat shock proteins with peptides and antigen presenting cells: chaperoning of the innate and adaptive immune responses. Annu Rev Immunol 2002; 20: 395-425.

114. Tsan MF, Gao B. Heat shock proteins and immune system. J Leukoc Biol 2009; 85: 905-910.

115. Liu B, Dai J, Zheng H, Stoilova D, Sun S, Li Z. Cell surface expression of an endoplasmic reticulum resident heat shock protein gp96 triggers MyD88-dependent systemic autoimmune diseases. Proc Natl Acad Sci USA 2003; 100: 15824-15829.

116. Zheng H, Dai J, Stoilova D, Li Z. Cell surface targeting of heat shock protein gp 96 induces dendritic cell maturation and antitumor immunity. J Immunol 2001; 167: 6731-6735.

117. Multhoff G, Botzler C, Jennen L, Schmidt J, Ellwart J, Issels R. Heat shock protein 72 on tumor cells: a recognition structure for natural killer cells. J Immunol 1997; 158: $4341-4350$.

118. Gastpar R, Gross C, Rossbacher L, Ellwart J, Riegger J, Multhoff G. The cell surfacelocalized heat shock protein 70 epitope TKD induces migration and cytolytic activity selectively in human NK cells. J Immunol 2004; 172: 972-980.

119. Gross C, Hansch D, Gastpar R, Multhoff G. Interaction of heat shock protein 70 peptide with NK cells involves the NK receptor CD94. Biol Chem 2003; 384: 267-279.

120. Elsner L, Muppala V, Gehrmann M, Lozano J, Malzahn D, Bickeboller H et al. The heat shock protein HSP70 promotes mouse NK cell activity against tumors that express inducible NKG2D ligands. J Immunol 2007; 179: 5523-5533.

121. Gastpar R, Gehrmann M, Bausero MA, Asea A, Gross C, Schroeder JA et al. Heat shock protein 70 surface-positive tumor exosomes stimulate migratory and cytolytic activity of natural killer cells. Cancer Res 2005; 65: 5238-5247.

122. Lv LH, Wan YL, Lin Y, Zhang W, Yang M, Li GL et al. Anticancer drugs cause release of exosomes with heat shock proteins from human hepatocellular carcinoma cells that elicit effective natural killer cell antitumor responses in vitro. J Biol Chem 2012; 287: 15874-15885.
123. Hanahan D, Weinberg RA. Hallmarks of cancer: the next generation. Cell 2011; 144: 646-674.

124. Lavia P, Mileo AM, Giordano A, Paggi MG. Emerging roles of DNA tumor viruses in cell proliferation: new insights into genomic instability. Oncogene 2003; 22: 6508-6516.

125. Jung $H$, Hsiung B, Pestal K, Procyk E, Raulet DH. RAE-1 ligands for the NKG2D receptor are regulated by E2F transcription factors, which control cell cycle entry. J Exp Med 2012; 209: 2409-2422

126. Guo H, Ingolia NT, Weissman JS, Bartel DP. Mammalian microRNAs predominantly act to decrease target mRNA levels. Nature 2010; 466: 835-840.

127. Ambros V. The functions of animal microRNAs. Nature 2004; 431: 350-355.

128. Stern-Ginossar N, Gur C, Biton M, Horwitz E, Elboim M, Stanietsky N et al. Human microRNAs regulate stress-induced immune responses mediated by the receptor NKG2D. Nat Immunol 2008; 9: 1065-1073.

129. Tsukerman $P$, Stern-Ginossar N, Gur C, Glasner A, Nachmani D, Bauman $Y$ et al. MiR$10 \mathrm{~b}$ downregulates the stress-induced cell surface molecule MICB, a critical ligand for cancer cell recognition by natural killer cells. Cancer Res 2012; 72: 5463-5472.

130. Cho WC. OncomiRs: the discovery and progress of microRNAs in cancers. Mol Cancer 2007; 6: 60 .

131. Nice TJ, Coscoy L, Raulet DH. Posttranslational regulation of the NKG2D ligand Mult1 in response to cell stress. J Exp Med 2009; 206: 287-298.

132. Salih HR, Rammensee HG, Steinle A. Cutting edge: down-regulation of MICA on human tumors by proteolytic shedding. J Immunol 2002; 169: 4098-4102.

133. Doubrovina ES, Doubrovin MM, Vider E, Sisson RB, O'Reilly RJ, Dupont B et al. Evasion from NK cell immunity by MHC class I chain-related molecules expressing colon adenocarcinoma. J Immunol 2003; 171: 6891-6899.

134. Holdenrieder S, Stieber P, Peterfi A, Nagel D, Steinle A, Salih HR. Soluble MICB in malignant diseases: analysis of diagnostic significance and correlation with soluble MICA. Cancer Immunol Immunother 2006; 55: 1584-1589.

135. Holdenrieder S, Stieber P, Peterfi A, Nagel D, Steinle A, Salih HR. Soluble MICA in malignant diseases. Int J Cancer 2006; 118: 684-687.

136. Ganem NJ, Pellman D. Limiting the proliferation of polyploid cells. Cell 2007; 131: 437-440.

137. Senovilla L, Vitale I, Martins I, Tailler M, Pailleret C, Michaud M et al. An immunosurveillance mechanism controls cancer cell ploidy. Science 2012; 337: 1678-1684.

138. Gardai SJ, McPhillips KA, Frasch SC, Janssen WJ, Starefeldt A, Murphy-Ullrich JE et al. Cell-surface calreticulin initiates clearance of viable or apoptotic cells through transactivation of LRP on the phagocyte. Cell 2005; 123: 321-334.

139. Mattarollo SR, Loi S, Duret H, Ma Y, Zitvogel L, Smyth MJ. Pivotal role of innate and adaptive immunity in anthracycline chemotherapy of established tumors. Cancer Res 2011; 71: 4809-4820

140. Zitvogel L, Apetoh L, Ghiringhelli F, Andre F, Tesniere A, Kroemer G. The anticancer immune response: indispensable for therapeutic success? J Clin Investig 2008; 118: 1991-2001.

141. Obeid M, Tesniere A, Ghiringhelli F, Fimia GM, Apetoh L, Perfettini JL et al. Calreticulin exposure dictates the immunogenicity of cancer cell death. Nat Med 2007; 13 54-61.

142. Ghiringhelli F, Apetoh L, Tesniere A, Aymeric L, Ma Y, Ortiz C et al. Activation of the NLRP3 inflammasome in dendritic cells induces IL-1beta-dependent adaptive immunity against tumors. Nat Med 2009; 15: 1170-1178.

143. Ma Y, Aymeric L, Locher C, Mattarollo SR, Delahaye NF, Pereira P et al. Contribution of IL-17-producing gamma delta T cells to the efficacy of anticancer chemotherapy. $J$ Exp Med 2011; 208: 491-503.

144. Fregni G, Perier A, Pittari G, Jacobelli S, Sastre X, Gervois N et al. Unique functional status of natural killer cells in metastatic stage IV melanoma patients and its modulation by chemotherapy. Clin Cancer Res 2011; 17: 2628-2637.

145. Hsu AK, Quach H, Tai T, Prince HM, Harrison SJ, Trapani JA et al. The immunostimulatory effect of lenalidomide on NK-cell function is profoundly inhibited by concurrent dexamethasone therapy. Blood 2011; 117: 1605-1613. 